$15^{\text {th }}$ International Conference on

AEROSPACE SCIENCES \& AVIATION TECHNOLOGY,

$\boldsymbol{A S A T}$ - 15 - May 28 - 30, 2013, Email: asat@mtc.edu.eg,

Military Technical College, Kobry Elkobbah, Cairo, Egypt,

Tel: +(202) 24025292-24036138, Fax: +(202) 22621908

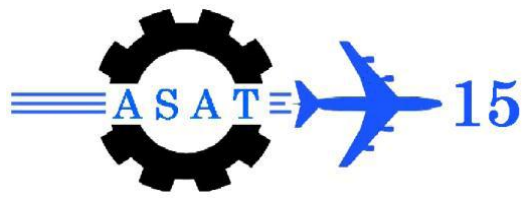

\title{
CFD Simulation of Fractal-Shaped Orifices for Flow Measurement
}

\section{Improvement}

\author{
A. Elsaey ${ }^{*}$, A. A. Aly ${ }^{\dagger}$ and M. Fouad
}

\begin{abstract}
The effect of using fractal-shaped orifice as flow meters for enhancing flow measuring techniques and their effect on pressure drop and flow mixing properties from point of view of losses saving and future designing of measuring devices with higher accuracy has been investigated.

In this frame, a numerical simulation has been used to study a turbulent flow through fractal-shaped orifices in pipes. Different fractal-shaped orifices; namely GEN-1, GEN-2, GEN-3 and GEN-4, have been constructed up to the forth iteration using a commercial CFD package. The studied pipe has a diameter of $0.14 \mathrm{~m}$ and a total length of $2.5 \mathrm{~m}$.

To validate the numerical simulation results, a direct comparison has been made with recently published experimental results for fractal-shaped orifice to illustrate the effect of the fractal-shaped on the pressure drop.

Numerical results showed that the fractal shaped orifice has a significant effect on the flow mixing properties and on the pressure drop downstream a pipe owing to their edge self-similarity shapes.
\end{abstract}

\section{Introduction}

Measuring the flow rate of liquids and gases in turbulent flow in piping systems is an important issue in many industrial applications which depend on accurate flow measurements. With most pressure differential flow rate measurement techniques, the flow rate is mostly determined by measuring the pressure drop across a certain measuring device in the pipe or duct. As an exploratory work, using fractal-shaped orifice as flow meters would have a significant effect on the pressure drop across these devices because of their edge self- similarity and hence their complex shape which enhance the mixing flow properties.

Here, a well-defined fractal-shaped orifice, of the Von Koch shape has been created with an increasing flow area up to the forth iterations and a case of ordinary circular orifice which area equals to the first iteration of the fractal shaped orifice namely GEN-1. Using numerical simulation, with the help of FLUENT commercial package [8] , the pressure drop across this fractal-shaped orifices and ordinary circular orifice set at different stations has been investigated and plotted.

\footnotetext{
* Faculty of Engineering, Cairo University, Cairo, Egypt.

${ }^{\dagger}$ Egyptian Armed Forces, Egypt, aboazm2008@yahoo.co.uk 
A validation of the numerical simulation results has been conducted by a direct comparison with recently published experimental results for Fractal-shaped orifices [1].

Regarding fractal objects, as in [2] , the scaling properties of the turbulent wake after different three-dimensional fractal grids in a wind tunnel has been investigated experimentally, as shown in Figure (1). They concluded that as the fractal dimension of the fractal grids increases the turbulent velocity fluctuations and become more equidistributed among scales, moreover the fractal turbulent wake is different from the bluff body turbulent wake as the fractal turbulent wake is longer. This may be explained because the increase of the turbulent intensity behind the grids.

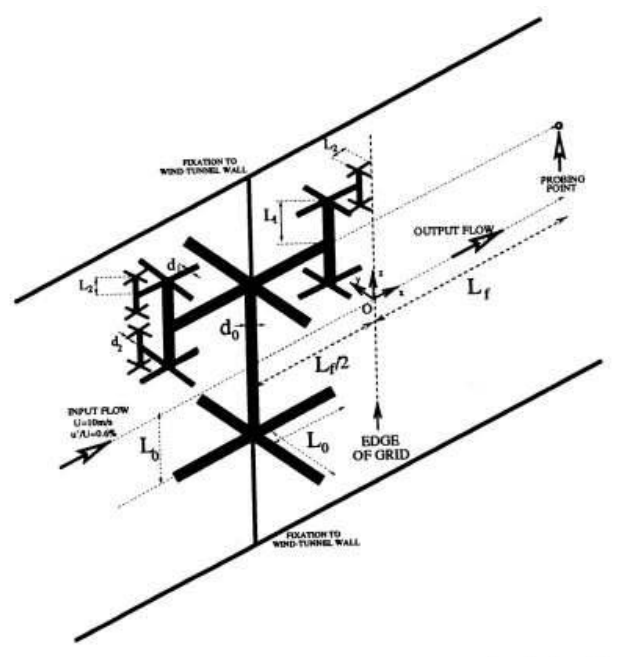

Fig. 1: Schematic representation of the 3D fractal grid [Queiros-Conde and Vassilicos (2000)]

Later in [3] a shell-model has been introduced to study the fractal-generated turbulent flow for different fractal dimension objects as in [2] they found that the power of the shell-model fractal forcing is an increasing function of the fractal dimension of the fractal object. Also, their results are sensitive to the modeling of the fractal forcing but supported the idea that the fractal forcing can be expected to change the turbulence scaling properties.

In [4] the scaling properties of objects having a fractal-shaped, self-similar structure and limited to the 4th iteration, has been investigated experimentally to study the turbulence generated in a wind tunnel flow using 10 hot wire anemometers at different positions downstream in the flow. They measured, as shown in Figure( 2), the energy spectrum and both the transverse and the longitudinal structure functions for different fractal objects and for different orientations and positions. They found that the scaling properties of the turbulence and the turbulence intensity depend on the orientation and the position of the fractal object relative to the velocity sensors. 


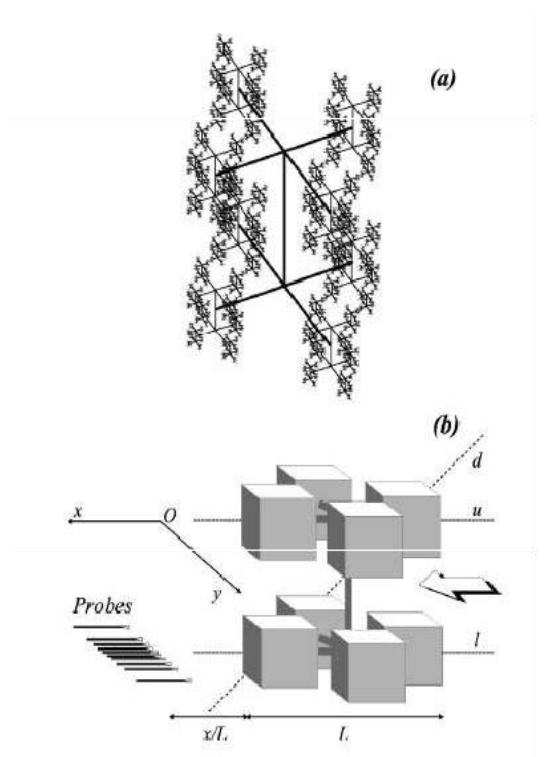

Fig. 2: (a) Schematic representation of the fractal-shaped objects. (b) Fractal-shaped Objects and the measurement configurations. [Staicu el al (2003)]

Later in [5] three different families of the fractal-generated turbulent flows generated in two different wind tunnels have been investigated experimentally. They investigated the dependence of the generated turbulent flows on the fractal geometry of the grid and they found that these flows are strongly dependent on the grid features; with small blockage ratio, the fractal grids generate turbulence with higher intensities and greater Reynolds numbers.

They also addressed some questions as to how turbulence decays when it is generated from many different sizes as produced in grid turbulence and in solving that they measured the flow velocities in different location using hot wire anemometry. They concluded that the best homogeneity is found where the space-filling pattern is obtained and can be improved as a function of the thickness parameter; in addition the thickness parameter also enhances the turbulence intensity. It was also noted that the turbulence far from the grids decays exponentially.

For the current study, a numerical simulation is used to study a turbulent flow through fractalshaped orifices and ordinary orifices in pipes. Four different fractal-shaped orifices; namely GEN1, GEN-2, GEN-3 and GEN-4, have been constructed up to the forth iteration. And a case of ordinary circular orifice which area equals to the first iteration of the fractal shaped orifice GEN-1. The calculations were made to figure out the fractal-shaped orifices sides and their self-similarity with the iterations. The fractal-shaped orifices meshes as well as the fractal orifice side mesh have been illustrated in Figures (3) and (4).

The studied pipe has a cross-sectional area of $0.0154 \mathrm{~m} 2$ with a pipe diameter of $0.14 \mathrm{~m}$ and a total length of $2.5 \mathrm{~m}$. The pipe set up in a horizontal configuration, this pipe configuration is similar to the experimental study in [1].

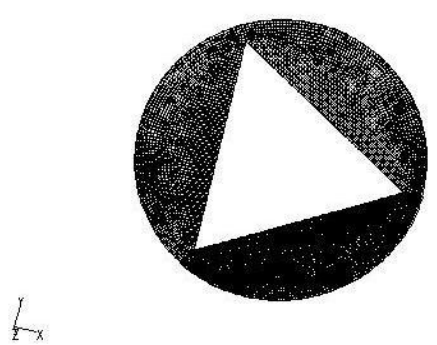

Figure 3: Fractal-orifice mesh, GEN-1. 


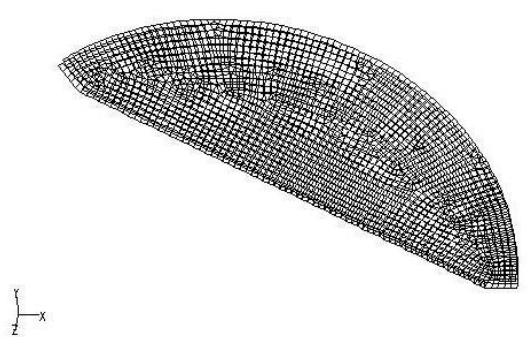

Figure 4: Fractal-orifice mesh, GEN-1, detailed pipe mesh near wall.

Four fractal-shaped orifices have been studied, first iteration of the fractal-shaped orifice, GEN-1, is with a self-similar triangular side of $108 \mathrm{~mm}$, and equivalent circular orifice diameter of $80.19 \mathrm{~mm}$. The second iteration of the fractal-shaped orifice, GEN-2, is with a self-similar triangular side of $36 \mathrm{~mm}$, and equivalent circular orifice diameter of $92.59 \mathrm{~mm}$. The third iteration of the fractal-shaped orifice, GEN-3, is with a self-similar triangular side of $12 \mathrm{~mm}$, and equivalent circular orifice diameter of $97.61 \mathrm{~mm}$. The last iteration of the fractalshaped orifice, GEN-4, is with a self-similar triangular side of $4 \mathrm{~mm}$, and equivalent circular orifice diameter of $99.75 \mathrm{~mm}$.

The four iterations for the fractal-shaped orifices and the equivalent circular orifices are described in Figures (5) and (6).
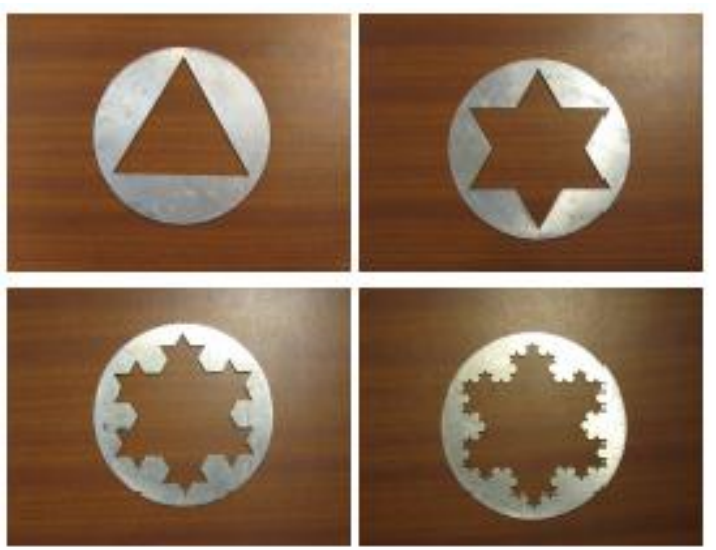

Figure 5: Fractal-shaped orifices for increasing flow area; first iteration GEN-1 (up left), second iteration GEN-2 (up right), third iteration GEN-3 (bottom left) and fourth iteration GEN-4 (bottom right).
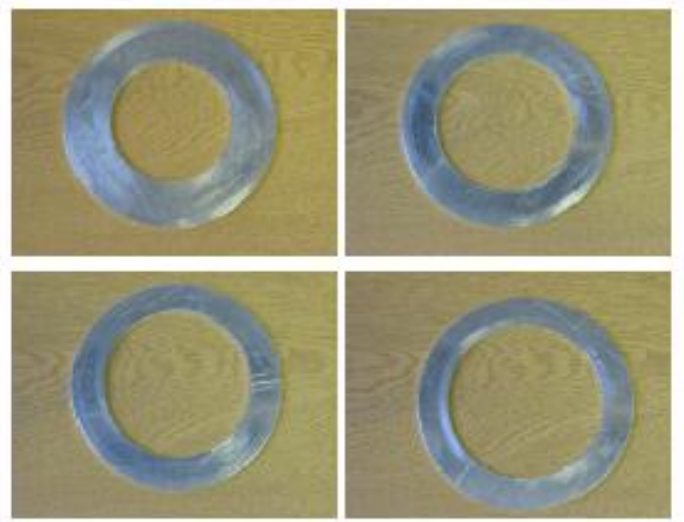

Figure 6: Circular orifices with equivalent area to the fractal-shaped orifices for increasing flow area; first iteration OR-1 (up left), second iteration OR-2 (up right), third iteration OR-3 (bottom left) and fourth iteration OR-4 (bottom right). 
The studied computational domain pipe was discretized using 3D cells along the longitudinal direction of the pipe using unstructured cells of the pipe geometry, as shown in Figure 7.

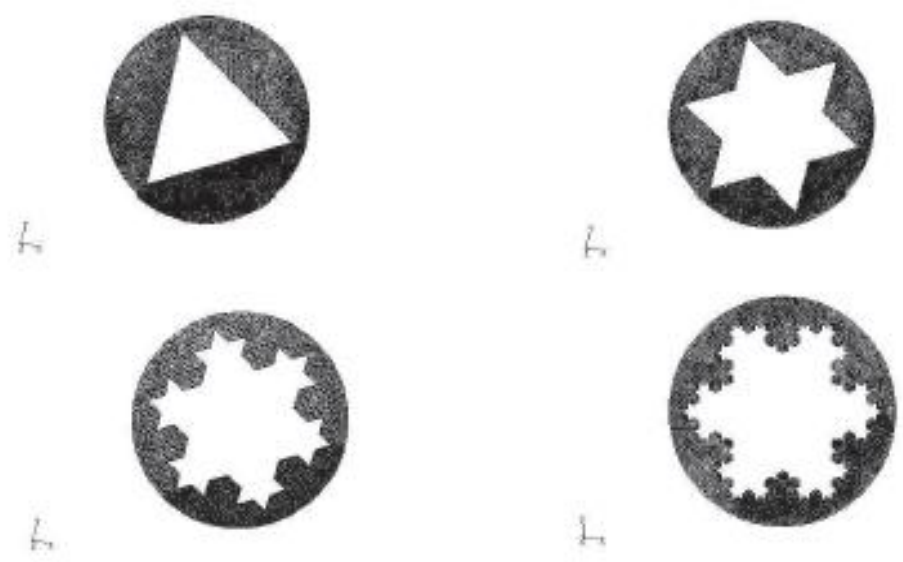

Figure 7: Fractal-shaped orifices pipe mesh for increasing flow area; first iteration GEN-1 (up left), second iteration GEN-2 (up right), third iteration GEN-3 (bottom left) and fourth iteration GEN-4 (bottom right).

Different zones with refined meshes were also introduced where required; around the fractalshaped orifice locations and near walls where high velocity gradients were expected to enhance the accuracy of the solution.

\section{Grid Sensitivity Analysis}

Different grid sizes for the studied 3-D geometry, GEN-1, have been investigated to check the solution dependency on this grid size, namely grid A, grid B, Grid C and Grid D. The parameters of each grid are shown in Table (1).

Table 1: Grid sensitivity analysis for different mesh cases, Case: GEN-1.

\begin{tabular}{c||c|c}
\hline \hline Grid name & $\begin{array}{c}\text { Total number of cells } \\
\text { ( hexahedral cell) }\end{array}$ & $\begin{array}{c}\text { Centre line velocity } \\
\text { point after } \mathbf{1 ~ D ~}(\mathbf{m} / \mathbf{s})\end{array}$ \\
\hline Grid A & 911840 & 6.344 \\
\hline Grid B & 1102140 & 6.348 \\
\hline Grid C & 1219440 & 6.351 \\
\hline Grid D & 1721137 & 6.352 \\
\hline \hline
\end{tabular}

The interested point has been selected to be after the fractal-shaped orifice where there is a high velocity gradient. This point has been assigned to determine the grid size at which no dependence of the numerical solution on the grid size is noticed. Results shown for grid D is chosen as the computational domain used in the present paper.

\section{Governing Equations}

The Reynolds-averaged Navier-Stokes (RANS) equations as in Equations (1) and (2) represent and taking the ensemble average yields the ensemble-averaged momentum equations .

For steady state case, they can be written in Cartesian tensor form as: 


$$
\begin{gathered}
\frac{\partial}{\partial x_{j}}\left(\rho \overline{u_{j}}\right)=S_{m} \\
\frac{\partial}{\partial x_{j}}\left(\rho \bar{u}_{i} \bar{u}_{j}\right)=-\frac{\partial \bar{p}}{\partial x_{j}}+\rho \vec{\beta}+\frac{\partial}{\partial x_{j}}\left[\mu\left(\frac{\partial \overline{u_{i}}}{\partial x_{j}}+\frac{\partial \overline{u_{j}}}{\partial x_{i}}\right)-\frac{2}{3} \delta_{i j} \frac{\partial \overline{u_{k}}}{\partial x_{k}}\right]-\frac{\partial}{\partial x_{j}}\left(\overline{\rho u_{i}^{\prime} u_{j}^{\prime}}\right)
\end{gathered}
$$

where $S_{m}$ is a mass source term, $\vec{\beta}$ is the body force per unit volume, $\rho$ and $\mu$ are the fluid density and viscosity respectively. $\delta_{\mathrm{ij}}$ is the Kronecker delta function $\left(\delta_{\mathrm{ij}}=1\right.$ if $\mathrm{i}=\mathrm{j}$ and $\delta_{\mathrm{ij}}=0$ if $\mathrm{i} \neq \mathrm{j}$ ).

Additional terms now appear that represent the effects of turbulence. This term is called Reynolds stresses, $\overline{\rho u_{i}^{\prime} u_{j}^{\prime}}$, which must be modeled in order to close the system of equations.

In the present study, k- $\varepsilon$ model will be used [7] to model turbulent flow. Attention should be paid to make these models suitable for wall-bounded flows. The value of $y^{+}$is checked for the pipe walls, orifice wall and orifice side wall, for fractal shaped orifice.

\section{Boundary Conditions}

Velocity inlet boundary condition is used to define the velocity profile at the inlet port; the inlet velocity was assigned to be a fully developed velocity profile at this inlet. This inlet velocity profile was assigned to match the boundary conditions in [1] to have a $R_{e}$ of 75000 based on the pipe diameter of value $8.3 \mathrm{~m} / \mathrm{s}$ for the averaged velocity and density of 1.15 $\mathrm{kg} / \mathrm{m}$ for working fluid of air. The turbulence is specified at the inlet port by turbulent intensity which is set to be $5 \%$ and hydraulic diameter which is the pipe diameter is set to be $0.14 \mathrm{~m}$. Outflow boundary condition is used to model flow exits where the details of the flow velocity and pressure are not known prior to solution of the flow problem. They are appropriate where the exit flow is close to a fully developed condition, as the outflow boundary condition assumes a zero normal gradient for all flow variables except for pressure, as shown in Figure (8).

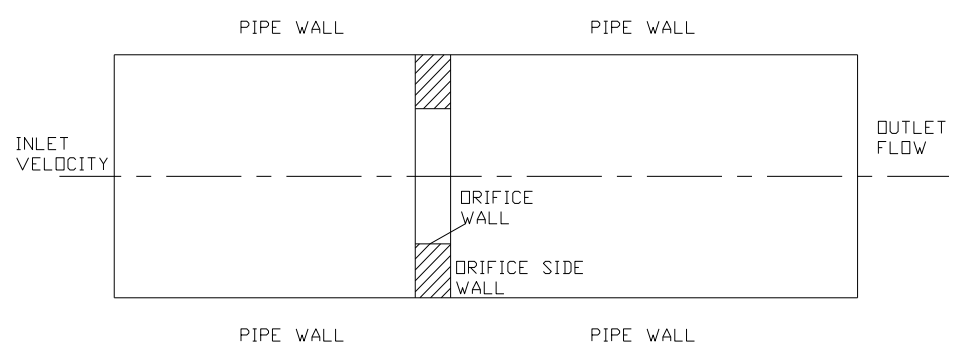

Figure 8: Boundary conditions used in numerical simulation.

Pipe wall was set as stationary walls. For the momentum equations, the no-slip condition is imposed on the solid surfaces. As indicated earlier, the standard wall functions are used to account for the effect of wall roughness on momentum. The symmetric planes were assigned for the self-similar of the fractal-shaped orifices and set as symmetry planes.

\section{Model Validation}

A direct comparison between numerical results and the previous published results to check the validity of the model presented in this study and to confirm some observations made earlier by other authors. Validation is carried out by calculating the pressure drop at stations configured in a previously published experimental work in [1]. The pressure drop has been calculated and presented per weighted area to represent the static pressure at that section. 
Also another comparison made between numerical simulation of fractal shaped orifice and another numerical simulation of ordinary orifice of area equivalent to fractal shaped orifice GEN-1 to show the effect of fractal shaped orifice over pressure drop across this devices as shown in Figures (9) and (10).

As shown in Figure(9), the pressure drop across fractal-shaped orifice resulted from the numerical simulation for GEN-1, and that obtained from the experimental results by [1]. The numerical simulation results are in a good agreement with that obtained from these experimental results, it has been noticed that the error between the numerical simulation results and the experimental results reduced as the distance downstream increased, initially after the orifice at $0.25 \mathrm{D}$ the error is about $5.4 \%$ and after $1 \mathrm{D}$ is about $1.25 \%$ which may be explained as that the existing of highly straining region may affect the numerical simulation results achieved.

As shown in Figure (10), the pressure drop resulted from the numerical simulation for fractalorifice GEN-1, and that obtained from the numerical simulation for ordinary orifice with the same flow area, it has been noticed that the pressure drop across the fractal shaped orifice is lower than that of ordinary orifice with the same flow area along the pipe after the orifice downstream the flow.

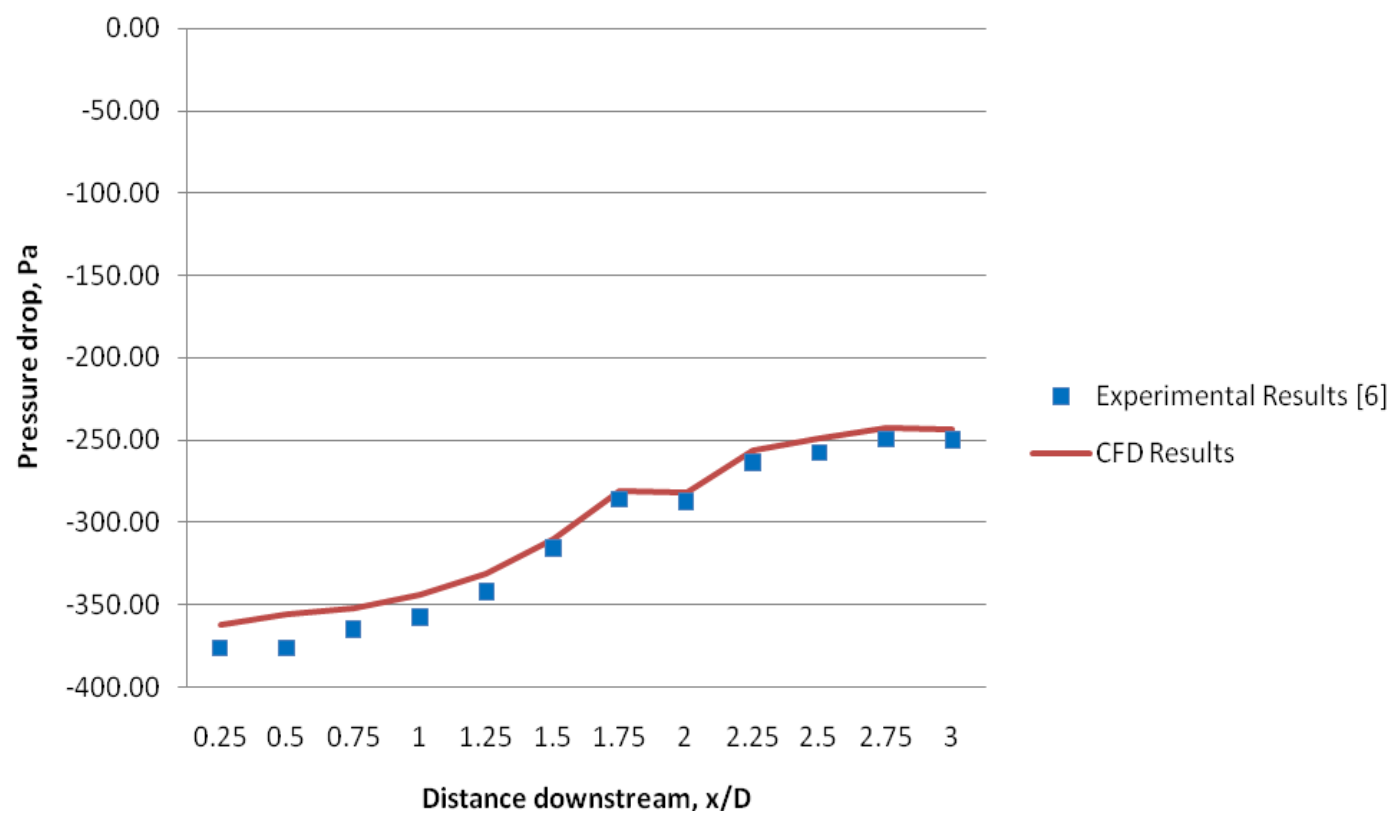

Figure 9: Comparison between pressure drop across fractal shaped orifices for the present study and ref. [1] results for fractal orifice generation GEN-1.

\section{CFD Results}

A detailed analysis for the numerical simulation results obtained for the fractal-shaped orifices namely, GEN-1, GEN-2, GEN-3 and GEN-4, in a turbulent pipe flow has been investigated. The results of the static pressure distributions and the axial velocity distributions along the pipe symmetrical plane downstream the fractal-shaped orifices for different drop generations of fractal-shaped orifices have been illustrated. The velocity contours as well as the pressure contours at different stations along the pipe centerline for different fractal-shaped orifices have been also introduced. 


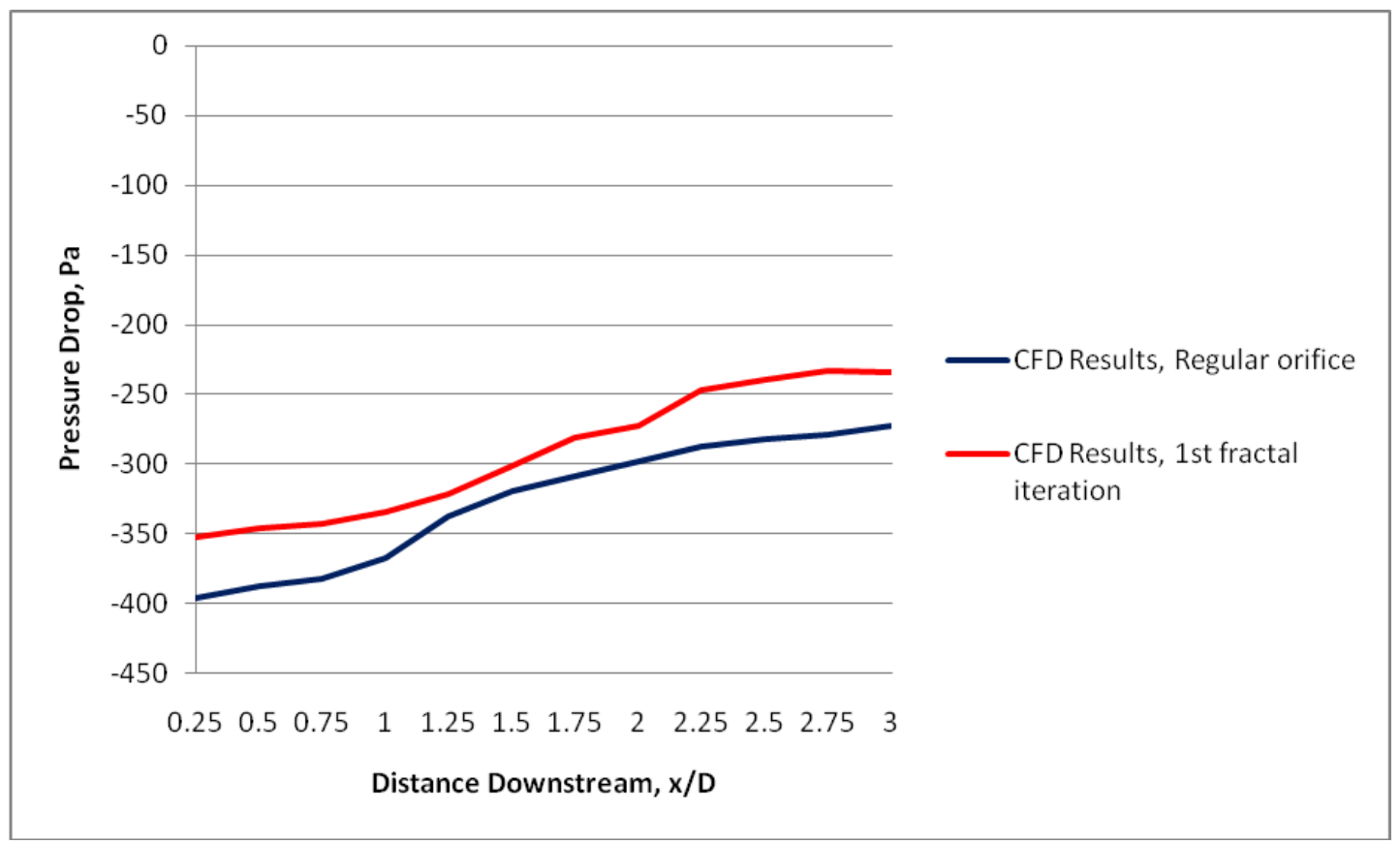

Figure 10: Comparison between pressure drops across numerical simulation of fractal shaped orifices GEN-1 and numerical simulation of ordinary orifice with the same flow area.

\subsection{Static Pressure Distributions along Symmetrical Pipe Plane}

The static pressure distributions along the pipe symmetrical plane have been introduced in Figures (11) to (14) for fractal-shaped orifice generations, GEN-1 to GEN-4 respectively. In addition, the static pressure distributions along the pipe symmetrical plane have been introduced in Figures (15) for ordinary orifice with the same flow area of fractal shaped orifice GEN-1.
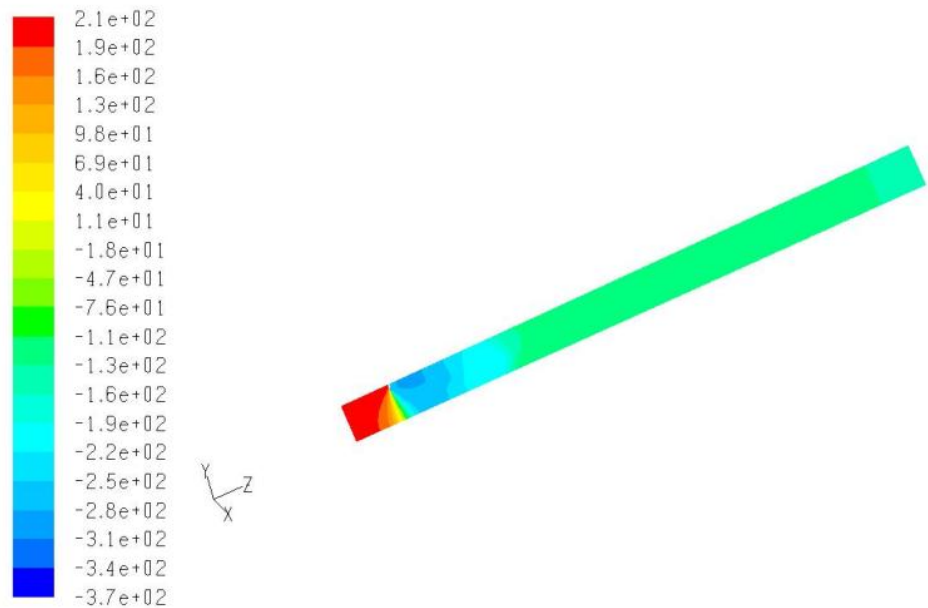

Fig 11: Static pressure contours for Fractal-shaped orifices pipe flow for GEN-1, at symmetric plane. 

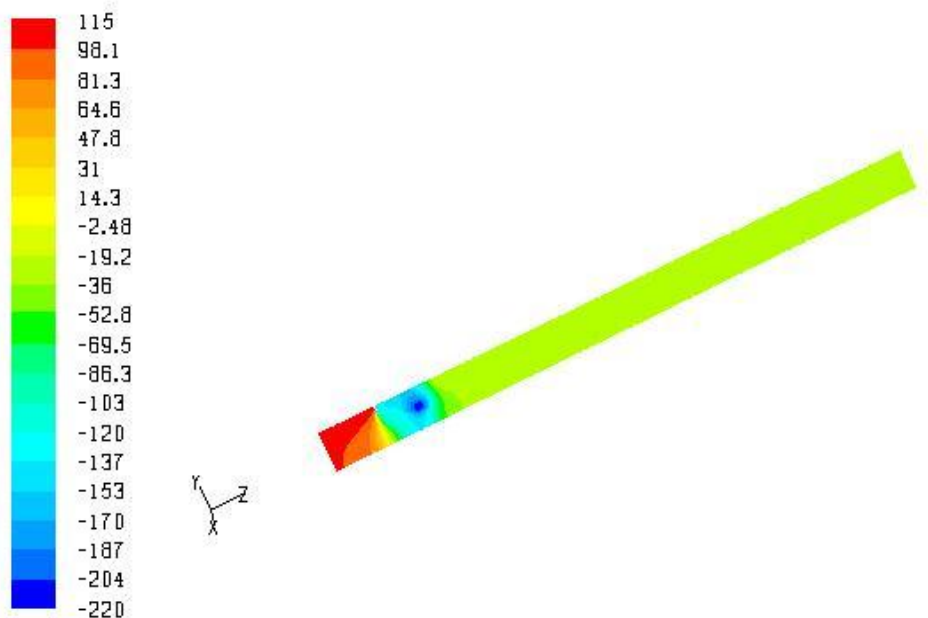

Fig 12: Static pressure contours for Fractal-shaped orifices pipe flow for GEN-2, at symmetric plane.
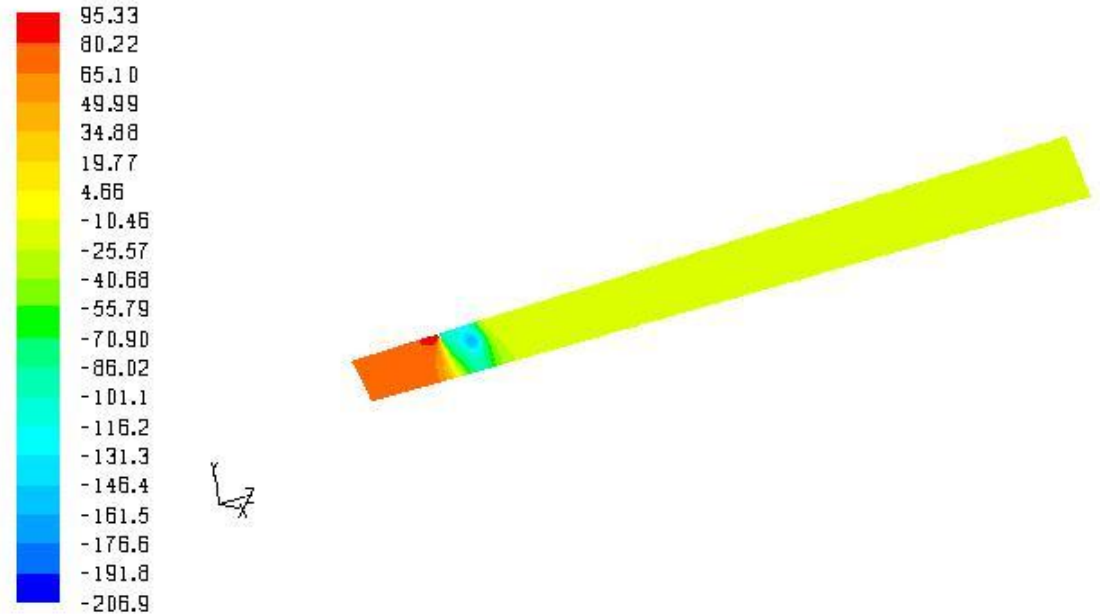

Fig 13: Static pressure contours for Fractal-shaped orifices pipe flow for GEN-3; at symmetric plane.

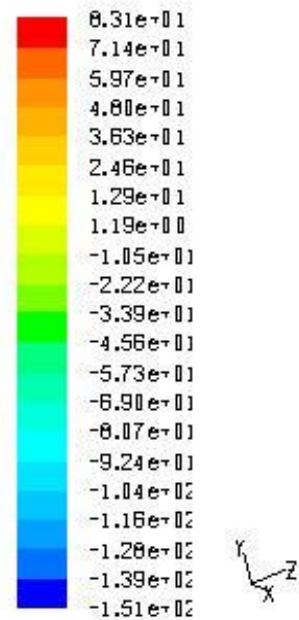

Fig 14: Static pressure contours for Fractal-shaped orifices pipe flow for GEN-4; at symmetric plane. 


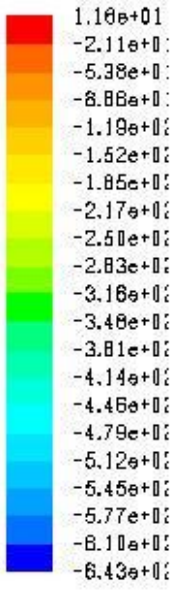

Fig 15: Static pressure contours for ordinary orifice pipe flow with flow area as fractal-shaped orifice GEN-1 ; at symmetric plane.

In these figures, it has been noticed that the pressure contours have a higher values for the first generation, GEN-1, in which the flow is more restricted and decreased with the increase of the fractal generation. And in comparison with the ordinary orifice with the same flow area equivalent to the fractal shaped orifice GEN-1 it's noticed that the pressure drop across the fractal shaped orifice GEN-1 is lower than that of ordinary orifice and flow recovery is faster and area affected zone is lower than that of ordinary orifice .

In addition, the pressure variation affected zones after the fractal-shaped orifices is decreased with the increase of the fractal-shaped generations, which may be explained as with the increase fractal-shaped generation, the geometrical properties of these self-similar shaped orifices had well affect the mixing zone and help the flow to be recovered faster than normal plain orifices. This point may help in designing a more sensitive flow meter for a short downstream length with good mixing properties.

In Figures (16) to (19), the static pressure values along the pipe centerline have been plotted for different fractal-shaped orifices, GEN-1 to GEN-4, while in Figure (20), it is for ordinary orifice of area equivalent to the area of fractal shaped orifice GEN-1.

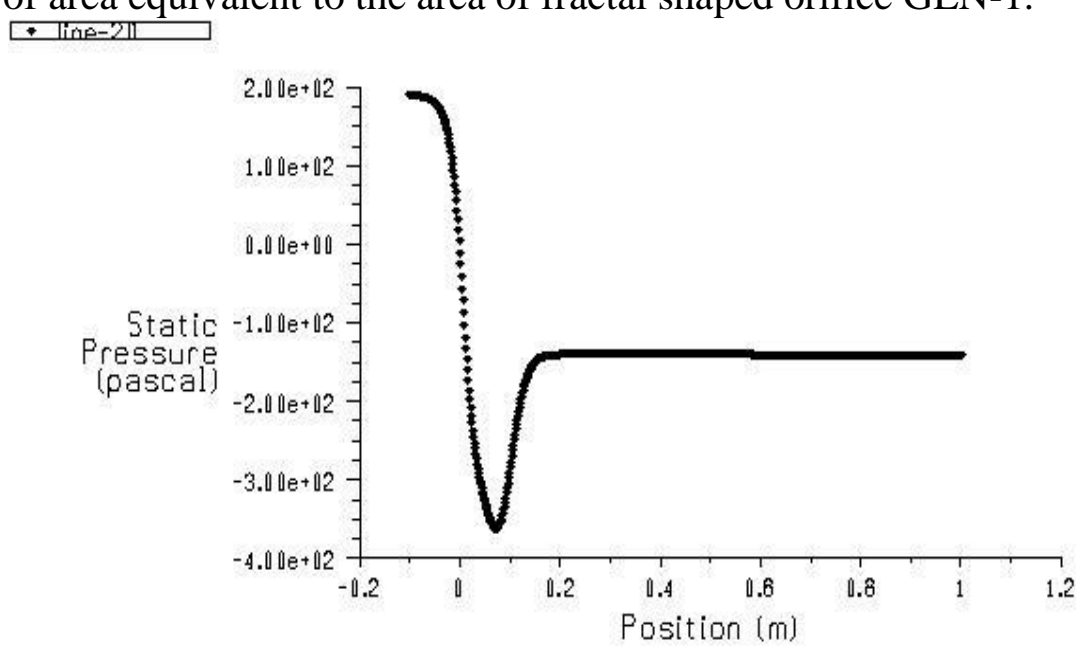

Figure 16: Static Pressure along the pipe centerline, results for generation GEN-1. 

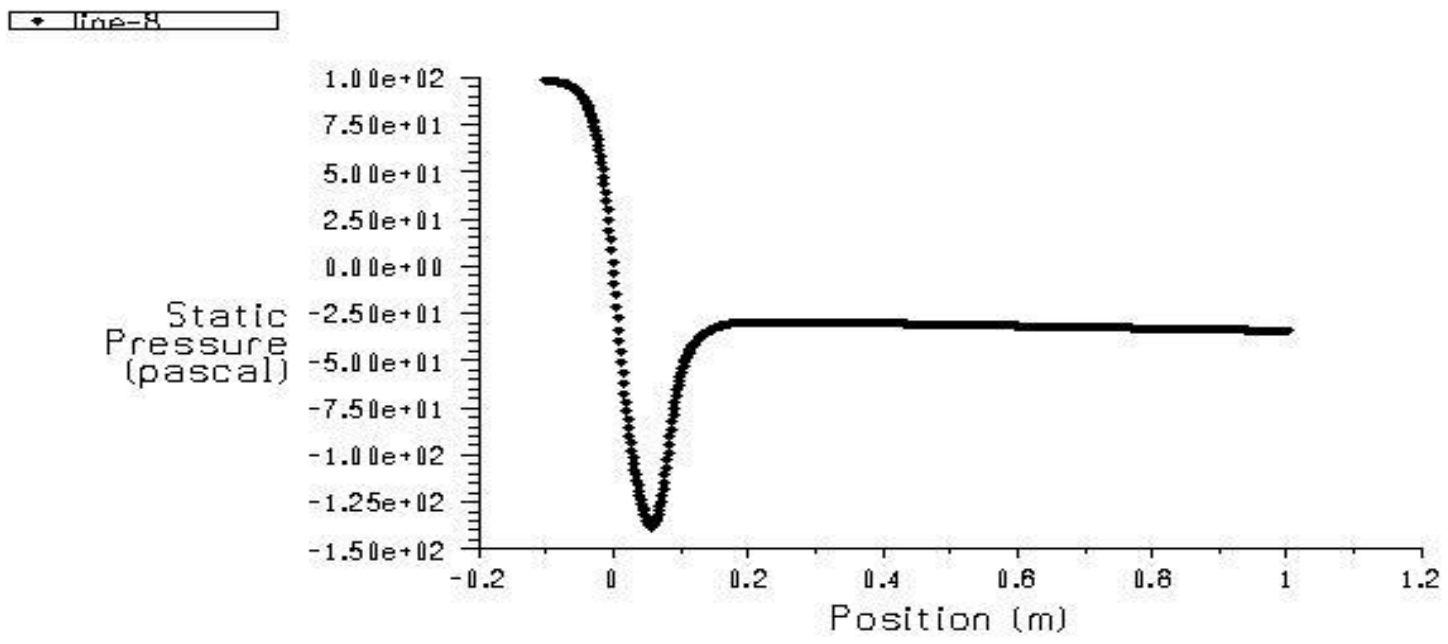

Figure 17: Static Pressure along the pipe centerline, results for generation GEN-2.

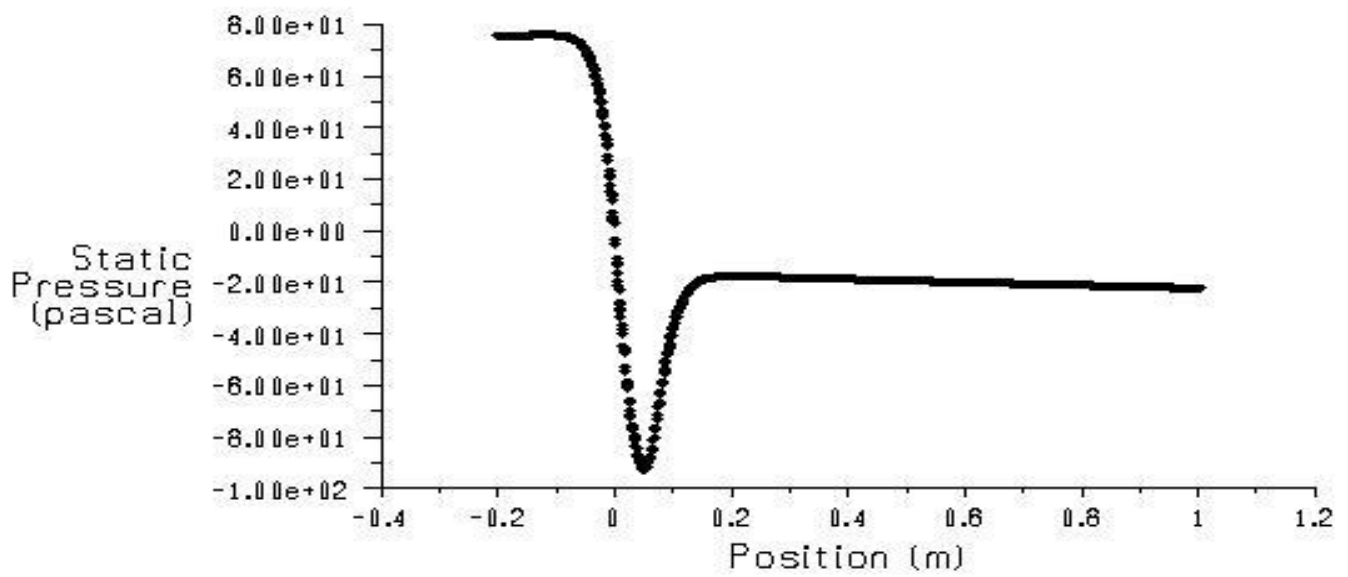

Figure 18: Static Pressure along the pipe centerline, results for generation GEN-3.

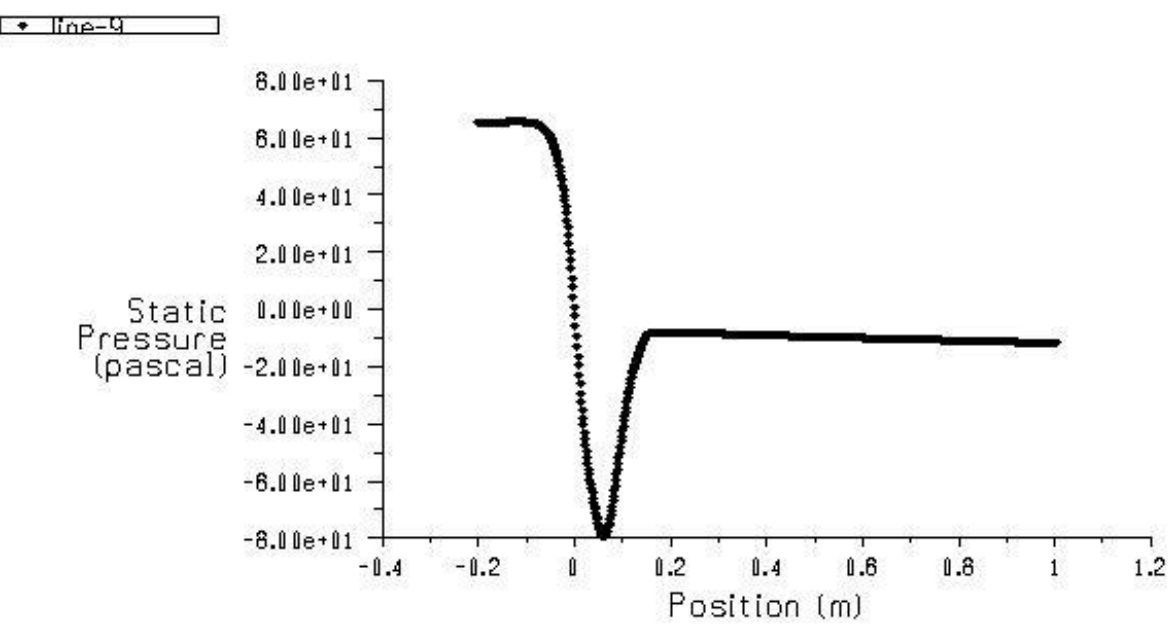

Figure 19: Static Pressure along the pipe centerline, results for generation GEN-4 


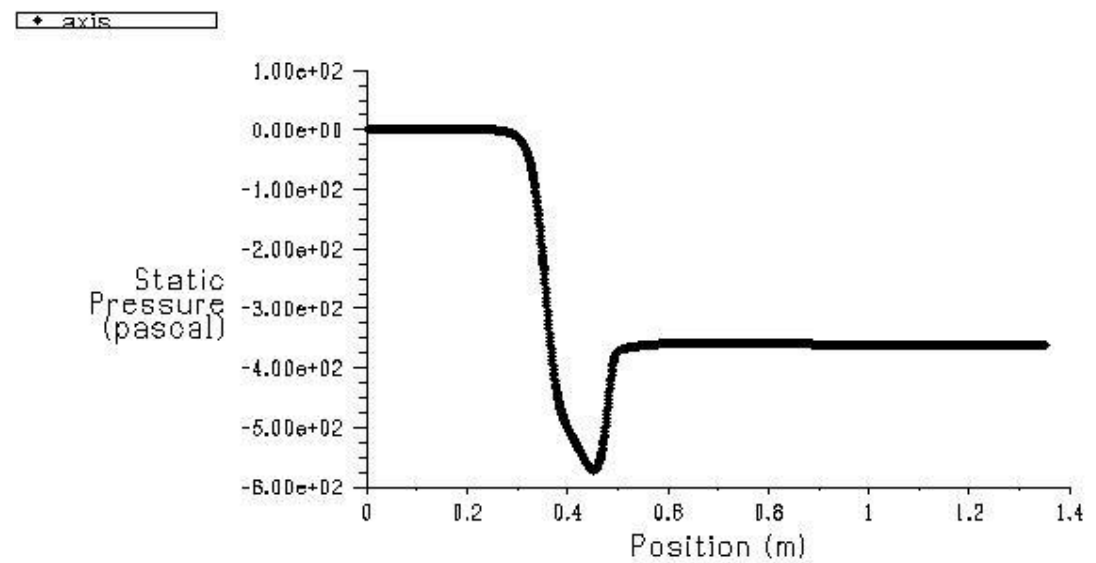

Fig. 20: Static Press along the pipe centerline, for ordinary orifice with area equivalent to GEN-1

In these figures, it has been noticed that the pressure drop across the fractal-shaped orifices have been decreased with the increase of the fractal generation, this relation has been plotted in Figure 21.

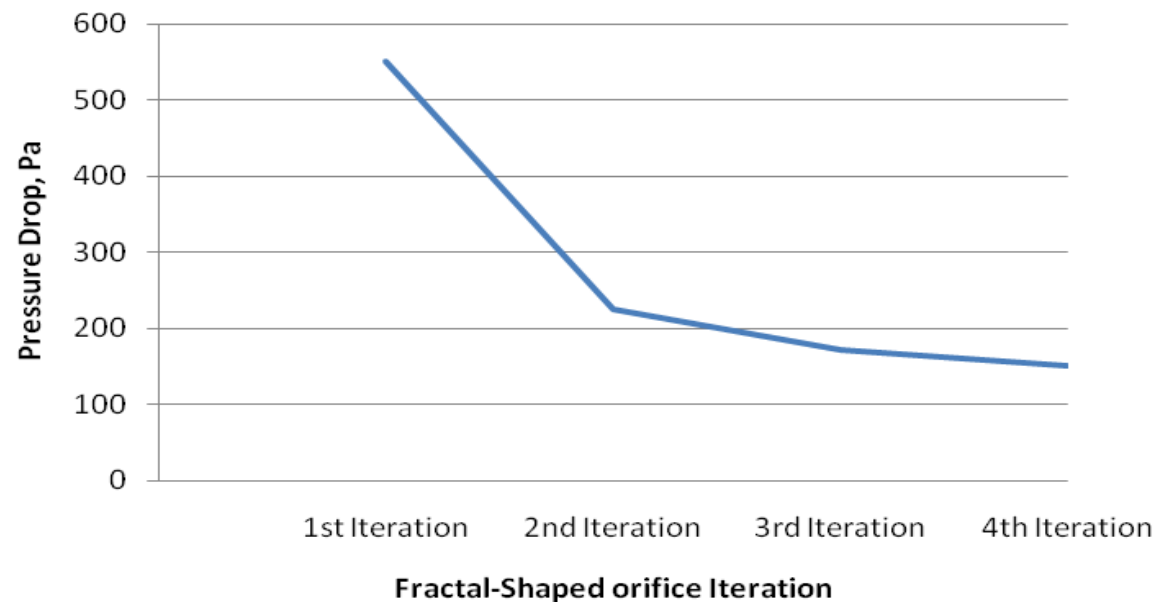

Figure 21: Pressure drop across fractal-shaped orifices as a function of the fractal iteration.

The use of the fractal-shaped orifice as a flow meter may lead to a lower pressure drop and reduced the losses across these orifices in comparison with ordinary orifice with the same flow area. The Pressure recovery across these fractal-shaped orifices has been illustrated to be reduced from that for the circular plain orifices as stated in [1], here the pressure recovery distance is plotted against the fractal-shaped orifices iterations, as shown in Figure (22), and it has been showed to be reduced with the increase of the fractal-shaped iteration which may lead to better use as of these fractal shaped as a flow meter. 


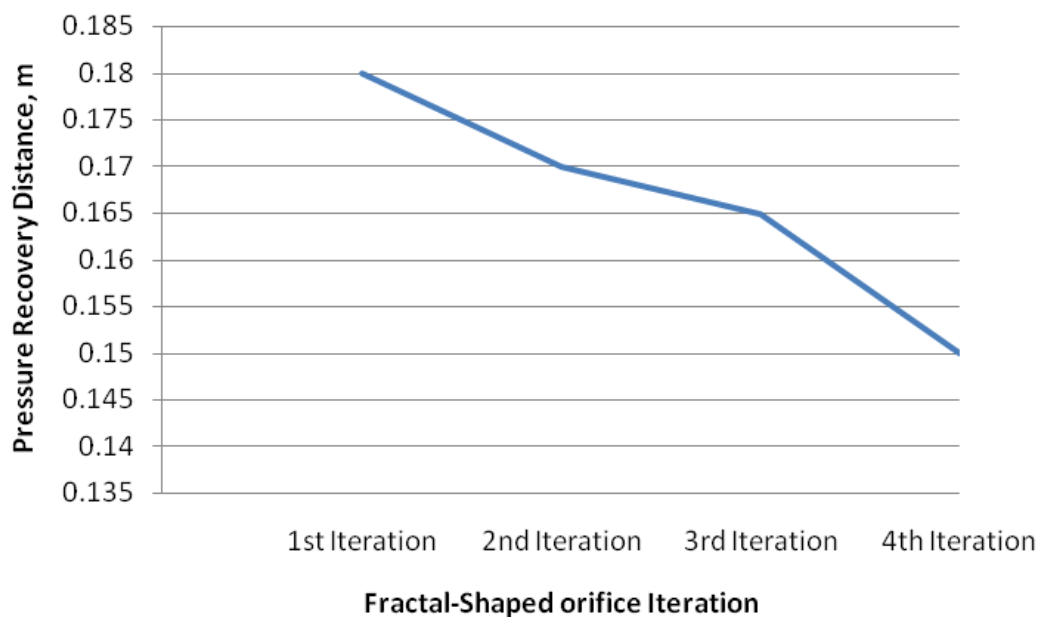

Fig 22: Press recovery distance across fractal orifices as a function of the fractal iteration.

\subsection{Axial Velocity Distributions along Symmetrical Pipe Plane}

The axial velocity distributions along the pipe symmetrical plane have been introduced in Figures (23) to (26) for fractal-shaped orifice generations, generation GEN-1 to generation GEN-4 respectively. And the axial velocity distributions along the pipe symmetrical plane have been introduced in Figure 27 for ordinary orifice with flow area equivalent to fractal shaped orifice GEN-1.
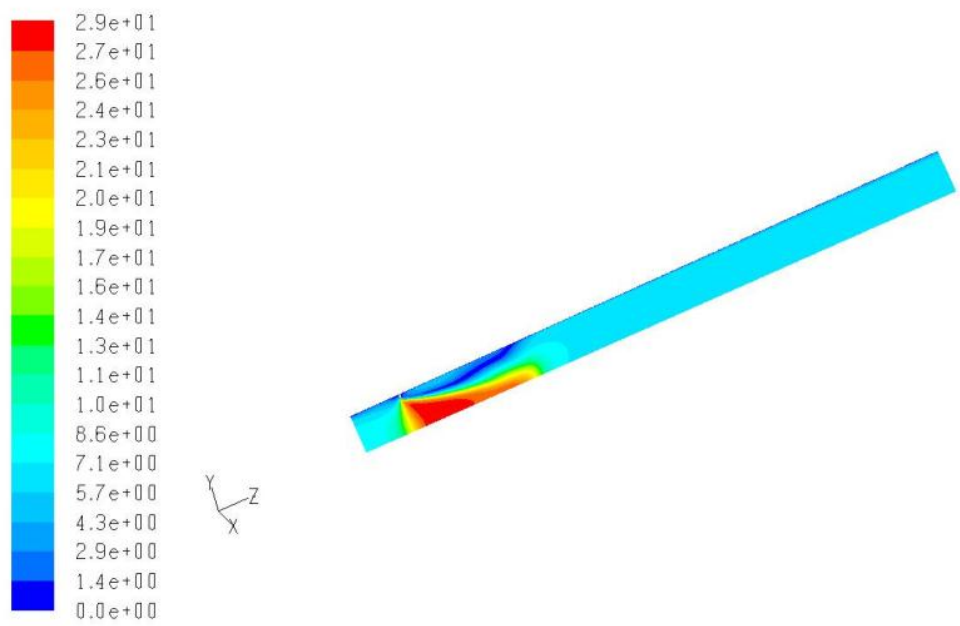

Fig 23: Axial Velocity contours for Fractal orifices pipe flow GEN-1; at symmetrical plane. 

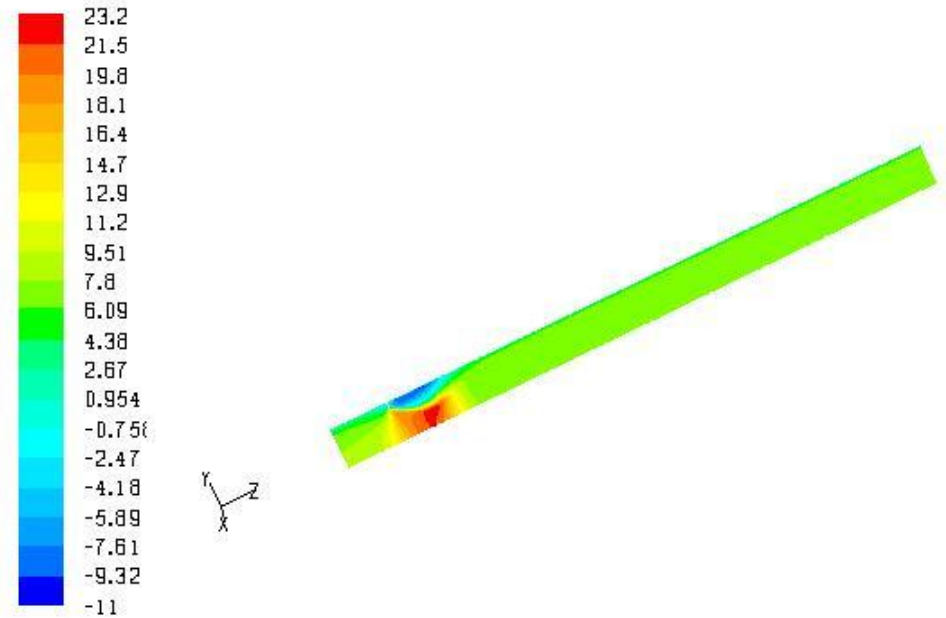

Fig 24: Axial Velocity contours for Fractal orifices pipe flow GEN-2; at symmetrical plane.
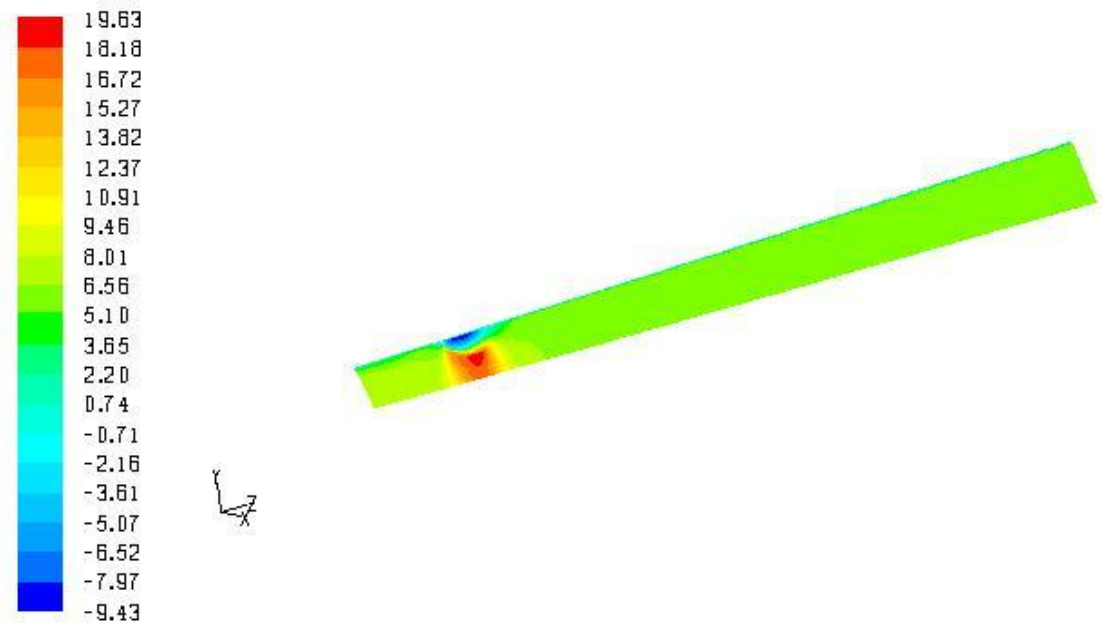

Fig 25: Axial Velocity contours for Fractal orifices pipe flow GEN-3; at symmetrical plane.
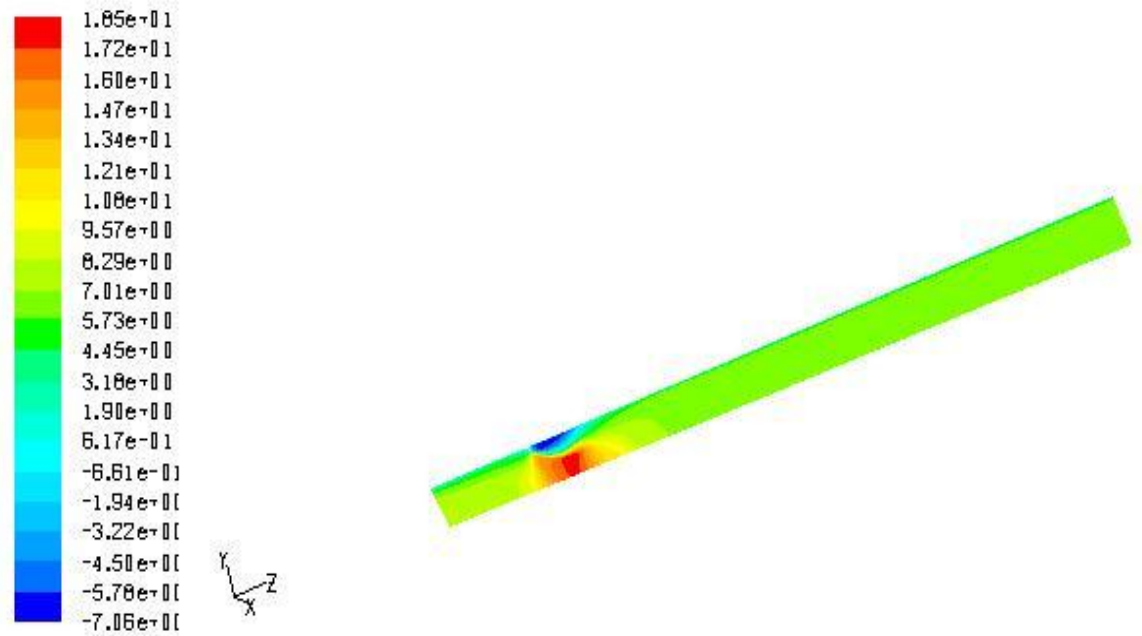

Fig 26: Axial Velocity contours for Fractal orifices pipe flow GEN-4; at symmetrical plane. 


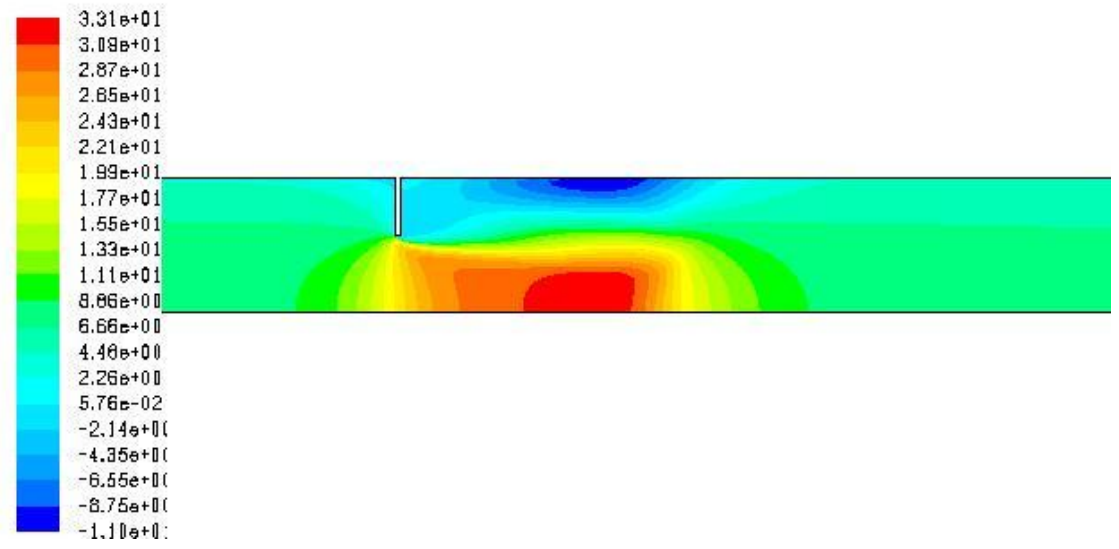

Figure 27: Axial velocity contours for ordinary orifice pipe flow with flow area equivalent to fractal shaped orifice generation GEN-1; symmetrical plane.

In these plots, with the increase of the fractal-shaped orifices iterations, the regions of the high velocity magnitude have been reduced compared with ordinary orifice with the same flow area of fractal shaped orifice GEN-1. This may explained as that with the increase of the fractal-shaped orifices iterations, the fractal-shaped orifices become smoother and uniform than the iteration before which may reflect on the velocity contours.

\subsection{Axial Velocity along Pipe Centerline}

The axial velocity along the pipe centerline has been introduced in Figures (28) and (29) for fractal-shaped orifice generations, generation GEN-1 and generation GEN-3 respectively. And for ordinary orifice with the same flow area equivalent to the fractal shaped orifice GEN-1 in Figure (30) and (31).

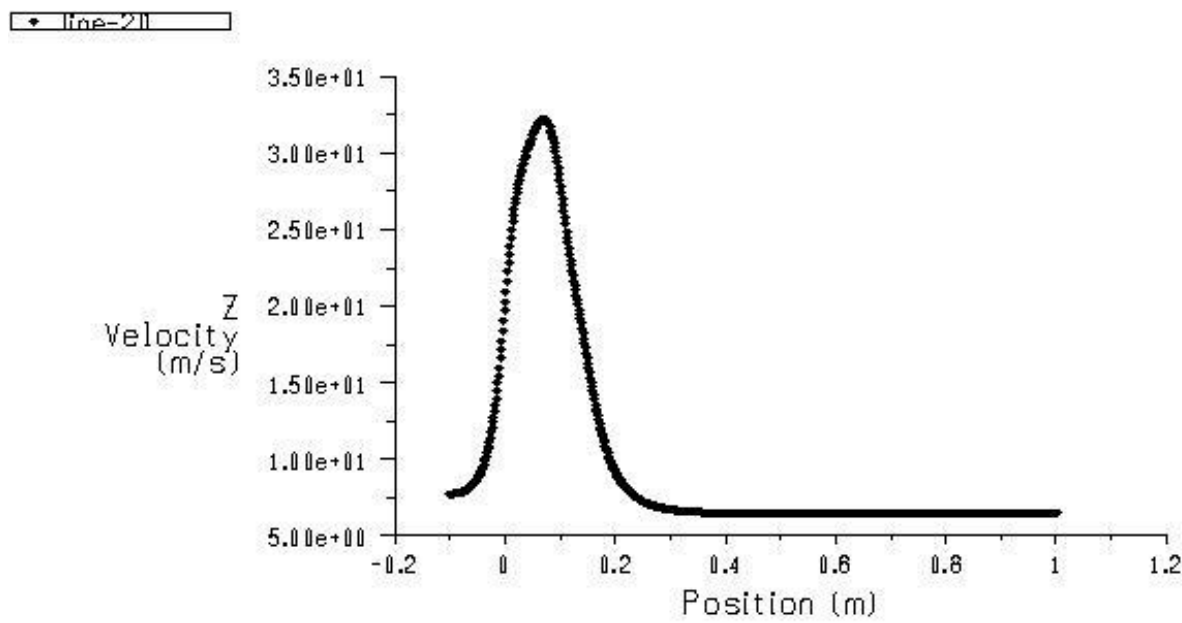

Figure 28: Axial Velocity along the pipe centerline, results for generation GEN-1. 


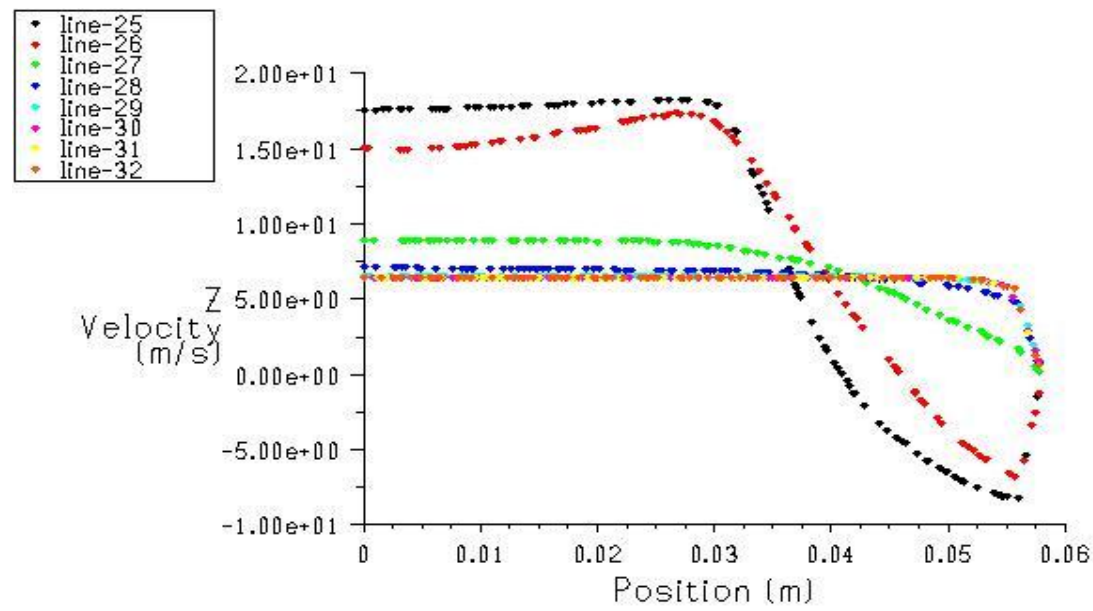

Figure 29: Axial velocity for Fractal-shaped orifices pipe flow for generation GEN-3; Legend: line-25: at distance D/4, line-26: at distance D/2, line-27: at distance 3D/4 line28: at distance D, line-29: at distance 5D/4, line-30: at distance 3D/2 .

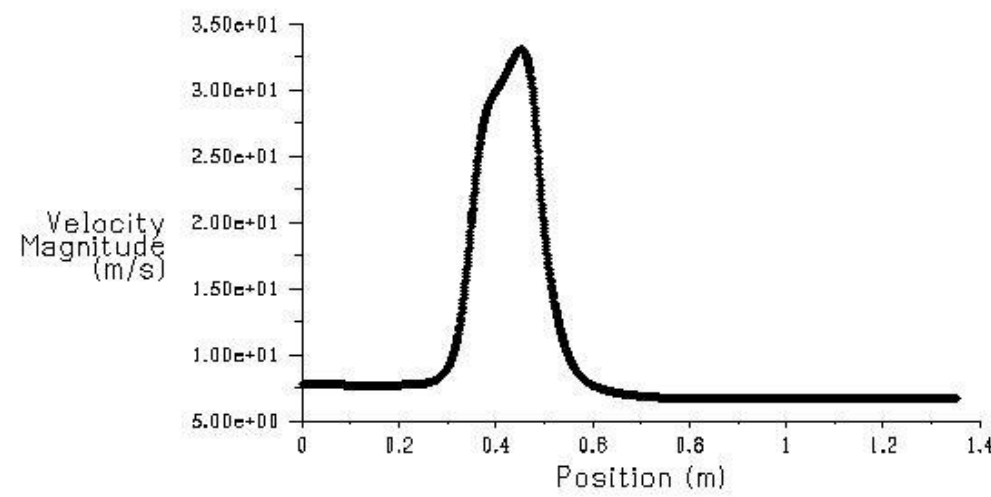

Velocity Magnitude Nov 21,2012
FLUENT 6.3 [axi, pbns, ske)

Fig 30: Axial velocity along pipe centerline for ordinary orifice pipe flow eqiv. to GEN-1

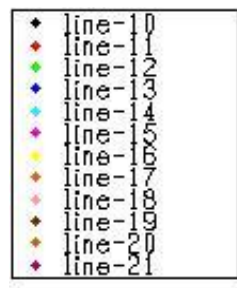

Velocity Magnitude

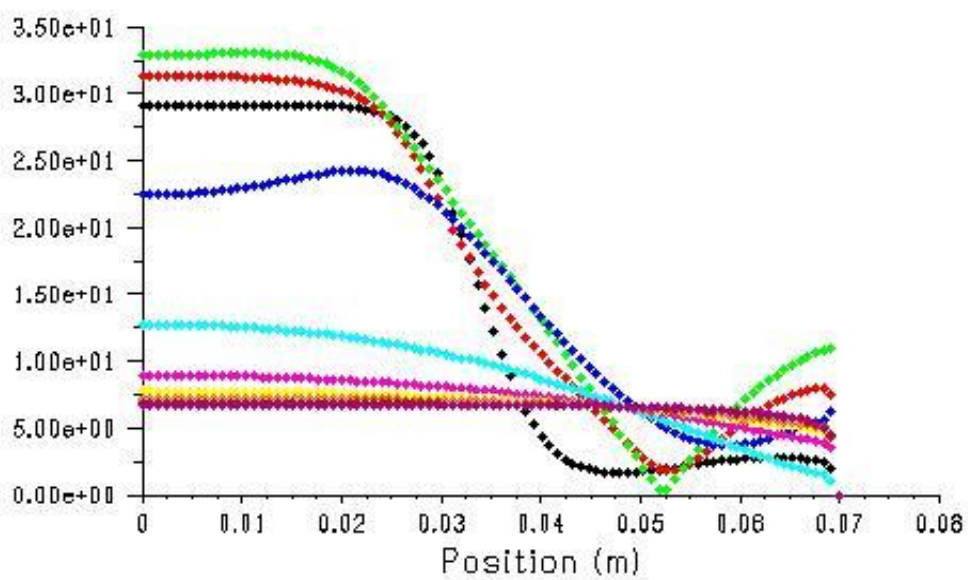

Fig 31:Axial velocity for ordinary orifice pipe flow equiv. to GEN-1;

Legend: line-10: at distance $\mathrm{D} / 4$, line-11: at distance $\mathrm{D} / 2$, line-12: at distance 3D/4, line-13: at distance $\mathrm{D}$, line-14: at distance $5 \mathrm{D} / 4$, line-15: at distance $3 \mathrm{D} / 2$, line-16: at distance 7D/4, line-17: at distance $2 \mathrm{D}$. 


\subsection{Pressure Contours at Different Pipe Stations}

The static pressure at station of $\mathrm{D} / 2$, for instance, after fractal-shaped orifices have been illustrated in Figures (32) to (35) for fractal-shaped orifice generation GEN-1 to GEN-4 respectively .
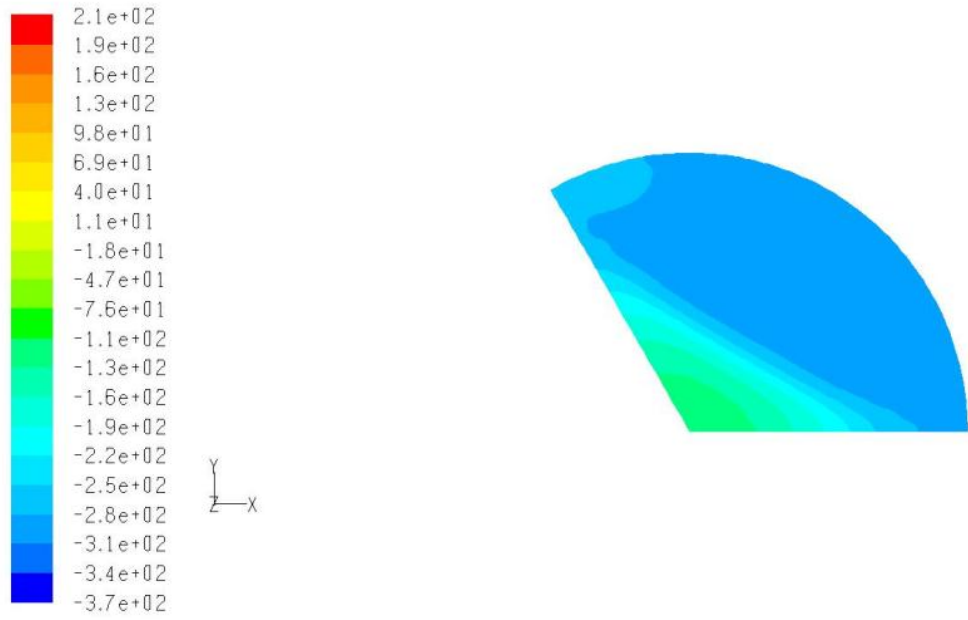

Fig 32: Static pressure contours for Fractal orifices pipe flow GEN-1; at distance D/2
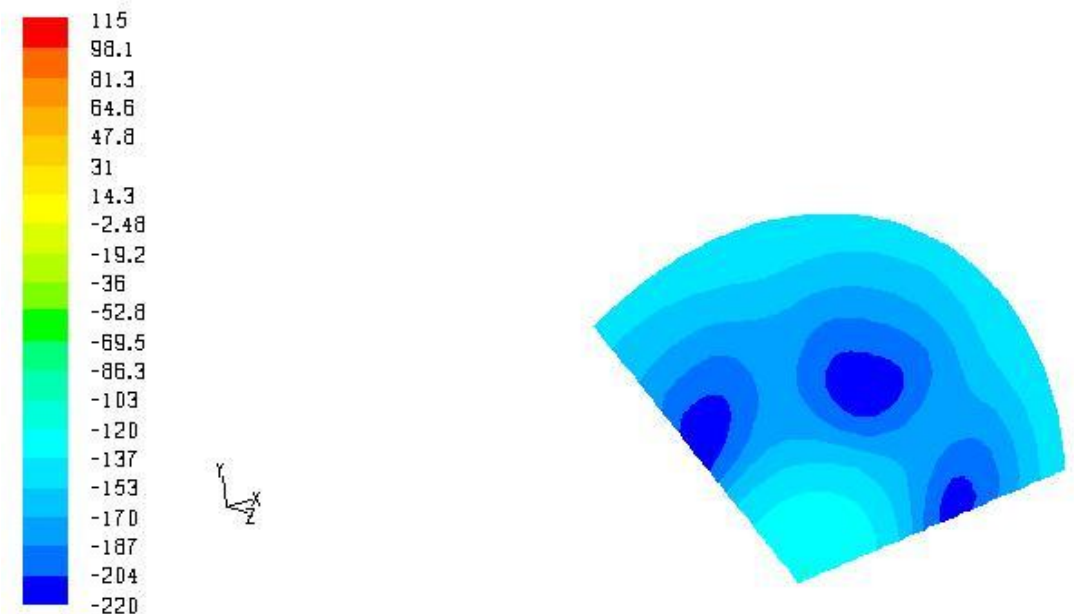

Fig 33: Static pressure contours for Fractal orifices pipe flow GEN-2; at distance D/2
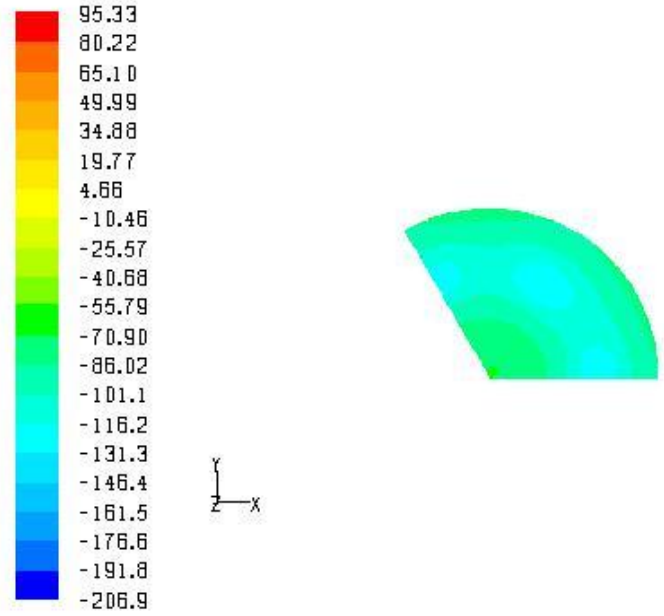

Fig 34: Static pressure contours for Fractal orifices pipe flow GEN-3; at distance D/2 . 

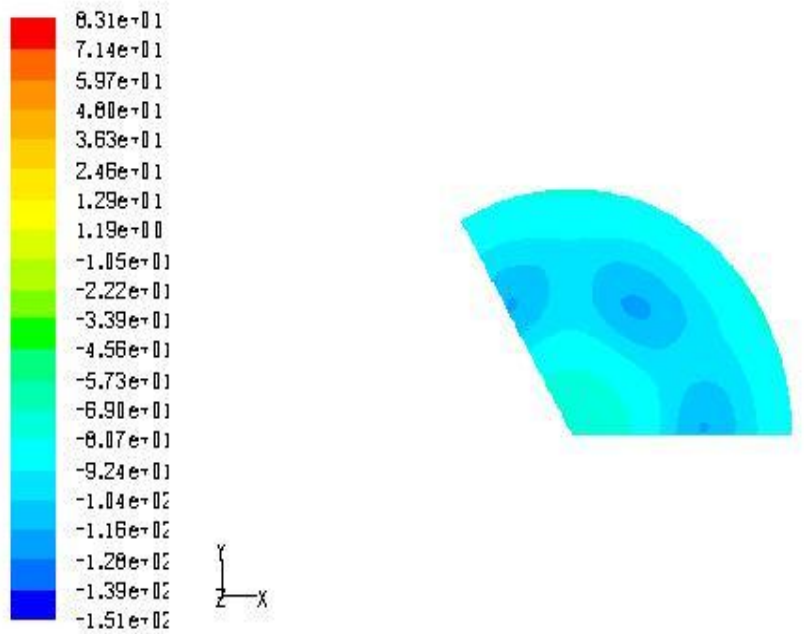

Fig 35: Static pressure contours for Fractal orifices pipe flow GEN-4; at distance D/2

\subsection{Axial Velocity Contours at Different Pipe Stations}

Here are some examples of the axial velocity contours at station of D/2 after fractal-shaped orifices that have been illustrated in Figures (36) to (39) for different fractal-shaped orifices generations, GEN-1 to GEN-4 respectively.
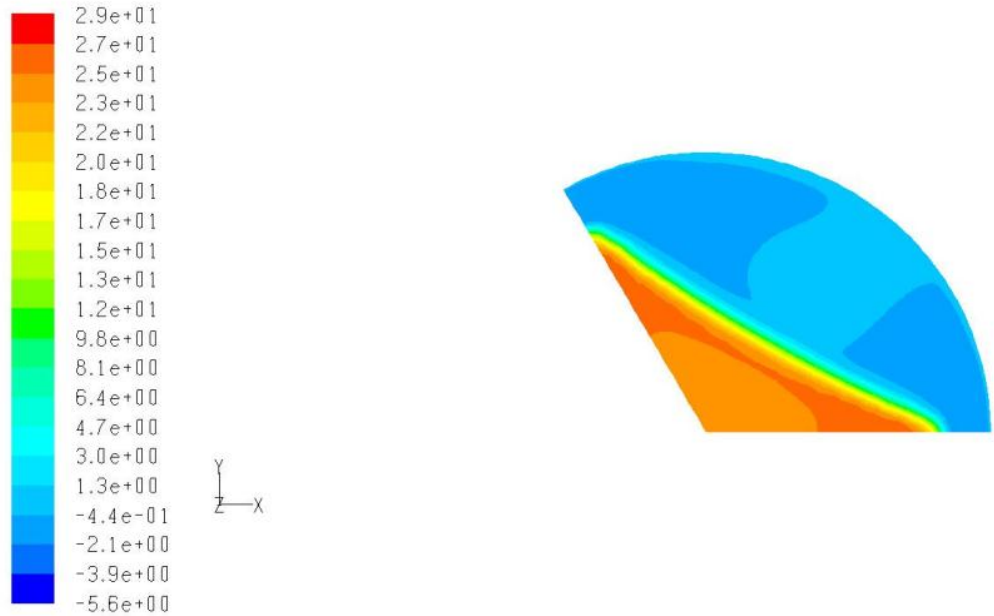

Figure 36: Axial velocity contours for Fractal orifices pipe flow GEN-1; at distance D/2.
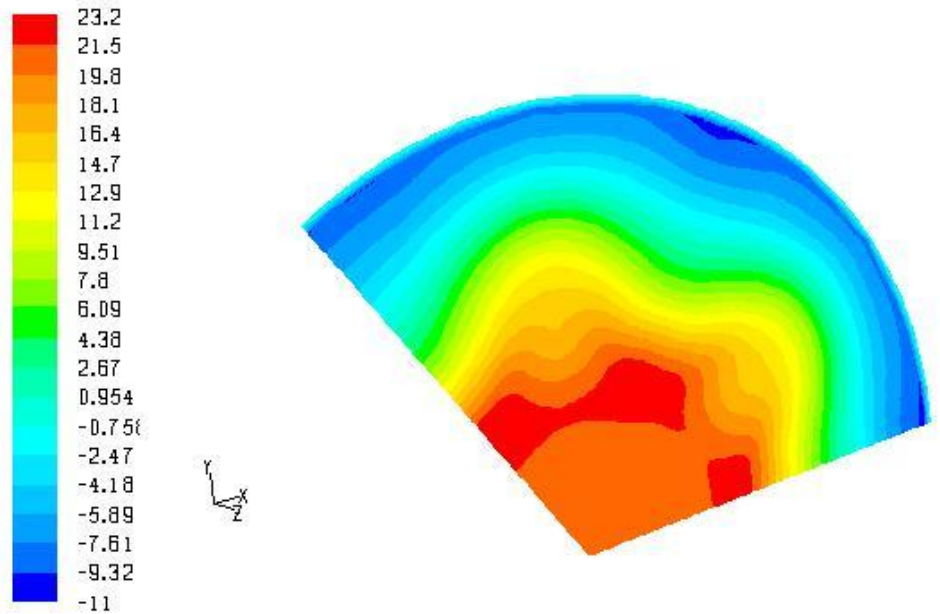

Figure 37: Axial velocity contours for Fractal orifices pipe flow GEN-2; at distance D/2 

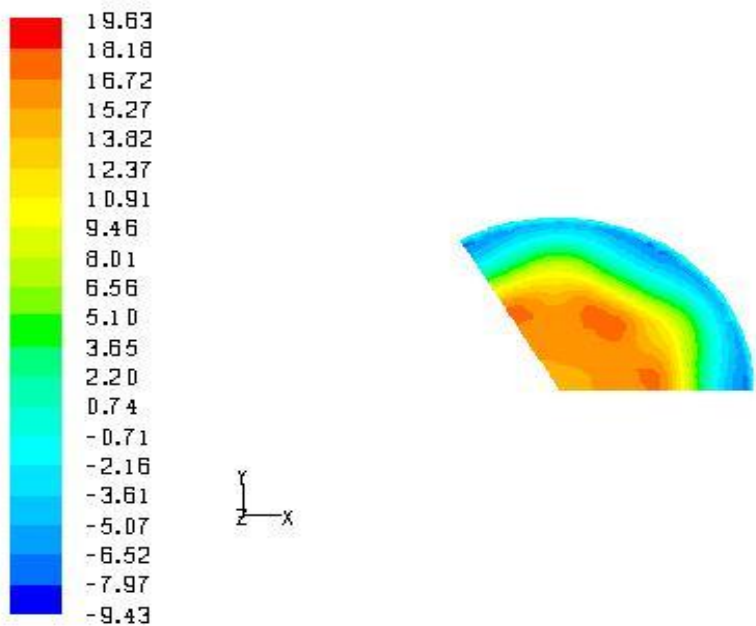

Figure 38: Axial velocity contours for Fractal orifices pipe flow GEN-3; at distance D/2

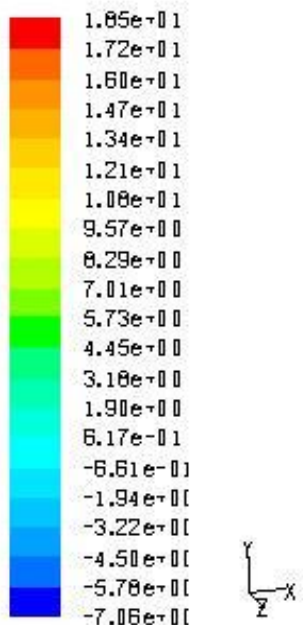

Figure 39: Axial velocity contours for Fractal orifices pipe flow GEN-4; at distance D/2

\section{Conclusions}

Study showed that the fractal shaped orifice has significant effect on the flow mixing properties downstream a pipe owing to their edge self-similarity shape also results show that the fractal-shaped orifices have a significant effect on the pressure drop. Furthermore, the pressure drop measured across the fractal-shaped orifices is found to be lower than that from regular circular orifices of the same flow areas.

The main thrust of the study is to evaluate the effect of using fractal shaped orifice as flow meters for enhancing flow measuring techniques and to illustrate the effect of fractal shaped orifice on pressure drop and flow mixing properties from point of view of losses saving and future designing of measuring devices with higher accuracy .

For the current study, a numerical simulation is used to study a turbulent flow through fractalshaped orifices and ordinary orifices in pipes. Different fractal-shaped orifices; namely GEN1, GEN-2, GEN-3 and GEN-4, have been constructed up to the forth iteration. And a case of ordinary circular orifice which area equals to the first iteration of the fractal shaped orifice namely GEN-1. The calculations were made up to figure out the fractal-shaped orifices sides and their self-similarity with the iterations.

The numerical simulation was made using a commercials CFD package; namely FLUENT, with its pre-processor GAMBIT. The studied pipe has a cross-sectional area of $0.0154 \mathrm{~m}^{2}$ with a pipe diameter of $0.14 \mathrm{~m}$ and a total length of $2.5 \mathrm{~m}$. To validate the numerical 
simulation results, a direct comparison have been made with recently published experimental.

The numerical simulation results are in a good agreement with that obtained from these experimental results, it has been noticed that the error between the numerical simulation results and the experimental results reduced as the distance downstream increased, initially after the orifice at $0.25 \mathrm{D}$ the error is about $5.4 \%$ and after $1 \mathrm{D}$ is about $1.25 \%$ which may be explained as that the existing of highly straining region may affect the numerical simulation results achieved and the simulation result almost coincide with that of experimental result as flow moves downstream the pipe as in figure (9).

In addition, a comparison with those results obtained from numerical simulation of ordinary orifices with the same flow area equivalent to fractal shaped orifice GEN-1 to illustrate the effect of the fractal-shaped on the pressure drop the comparison show that the pressure drop across the fractal shaped orifice is lower than that of ordinary orifice by $12.5 \%$ downstream pipe flow as in figure (10) and finally CFD is able to predict the effect of insertion fractal-shaped orifices in turbulent pipe flow.

It could conclude that; the numerical simulation results for the effects of the presence of the fractal shaped orifice over the pressure drop across this devices show a great coincide with the experimental results had been done for the same purpose. The numerical simulation results shows that the pressure drop across the fractal shaped orifice is lower than that of ordinary orifice with the same flow area by around $12.5 \%$ along the pipe downstream. The numerical simulation contours and plots shows that as the fractal iteration of the orifice increase there are better effect on the flow mixing which accelerate flow recovery downstream this devices. The numerical simulation shows that the range of velocity scales developed by the fractal orifices is broader than that with the ordinary orifice with the same flow area.

\section{References}

[1] Abou El-Azm, A., Chong, A., Nicolleau, F. and Beck, S. 2010 "Experimental Study of the pressure drop after fractal-shaped orifices in turbulent pipe flows", Experimental Thermal and Fluid Science, 34, 104-111.

[2] Queiros-Conde, D. and Vassilicos, J. 2000 "Intermittency in turbulence and other dynamical systems", Cambridge University Press, First Edition.

[3] Mazzi, B., Okkels, F. and Vassilicos, J. 2002 "A Shell-model approach to fractalinduced turbulence”, European Physical Journal B, 28, 243-251.

[4] Staicu, A., Mazzi, B., Vassilicos, J. and Van de Water, W 2003 "Turbulent wakes of fractal Objectes", Physical Review E, 67, 066306.

[5] Hurst, D. and J.C. Vassilicos, J. 2007 "Scalling and Decay of fractal-generated turbulence", Physics of Fluids, 19, 035103.

[6] Seoud, R. and Vassilicos, J. 2007 "Dissipation and decay of fractal-generated turbulence", Physics of Fluids, 19, 105108.

[7] Jones, W. P., and Launder, B. E., 1972 "The Prediction of Laminarization With a TwoEquation Model of Turbulence" Int. J. Heat Mass Transfer, Vol. 15, 301-314.

[8] FLUENT 6.2 Documentation, (C) Fluent Inc. 2005-01-04. 